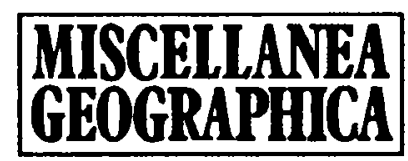

WARSZAWA 1994 Vol. 6

Andrzej Richling

\title{
L' ÉCOLOGIE DU PAYSAGE COMME SYNTHÈSE DES ÉTUDES SUR LE MILIEU NATUREL
}

Le terme "écologie du paysage" a été introduit par Troll à la fin des années trente. Selon lui, l'analyse du fonctionnement du contenu du paysage et l'explication de ses relations, multiples et qui se modifient, constitue le but principal de cette discipline (Troll 1950). Il soulignait l'importance de ces études pour la vie pratique, surtout pour la planification. Ainsi comprise, l'écologie du paysage est, d'après Vink (1983), le fruit du mariage entre la géographie et la biologie.

L'utilisation des photographies aériennes a stimulé le développement de l'écologie du paysage. Troll considérait que l'analyse de ces photographies fait partie de l'écologie du paysage même si elles servent aux archéologues ou aux pédologues. Le paysage géographique et le réseau écologique de cause à effet, existant dans ce paysage, constituent le but de l'analyse des photographies aériennes.

En 1963, Troll a modifié sa définition de l'écologie du paysage, se rapprochant de la conception de l'écosystème de Tansley. Il s'agit d'étudier le complexe des relations entre les biocénoses et leurs conditions naturelles dans des unités paysagiques définies (cf. Schreiber 1990). Certains biologistes englobaient l'écologie du paysage ainsi comprise dans les sciences biologiques (écologie pratiquée à l'échelle du paysage). Mais, comme écrit à juste titre Zonneveld (1990), l'écologie du paysage se rapporte à l'ensemble des phénomènes et concerne donc des spécialistes différents: géographes, biologistes, hydrologues, ainsi que les chercheurs, dont les techniciens, qui s'occupent des effets de l'activité humaine sur le milieu naturel.

Troll a traduit en anglais le terme "écologie du paysage" par "geoecology". Dans le monde anglo-saxon, mais aussi dans d'autres pays de l'Europe de l'Ouest, cette "géoécologie" (écologie du paysage) est très souvent comprise comme l'aménagement des ressources naturelles - et définie de manière pragmatique comme une science qui s'occupe de ce dont s'occupent les écologistes (Leser, Rodd 1991). Même si cela n'était pas formulé explicitement, les fondateurs et les membres de l'Association internationale de l'écologie du paysage (IALE) représentaient un point de 
vue similaire. Il est toujours exprimé par les praticiens, de diverses formations. Une telle formulation réduit néanmoins les définitions précédentes. Il est sans doute vrai que l'apparition de l'écologie du paysage est liée à la demande économique, mais les recherches appliquées ne peuvent pas se développer sans un fond théorique et méthodologique. Il est également à noter que dans les pays germaniques la géoécologie est considérée comme cette partie de l'écologie du paysage qui s'occupe du milieu abiotique, complémentaire à la bioécologie qui concerne le milieu biotique (Schreiber 1990).

La spécificité de l'écologie du paysage s'exprime dans une approche globale de la matière étudiée, c'est-à-dire du paysage, qui englobe également l'homme et les effets de son activité - et qui est analysée dans ses aspects structurels, fonctionnels et visuels. L'écologie du paysage embrasse l'analyse des éléments du paysage et des relations existant entre eux, l'identification des unités spatiales naturelles, leur classification hiérarchique et la valorisation des différentes dispositions du milieu naturel pour les différentes activités humaines - ainsi qu'un diagnostic sur la manière d'organiser l'espace naturel.

Selon Delcourt et Delcourt (1988) les buts principaux de l'écologie du paysage consistent à apprécier la disposition écologique du paysage et le déroulement des processus paysagiqus dans le temps, ainsi qu'à déterminer les conséquences écologiques de la transformation des paysages naturels (ou proches des paysages naturels) en des paysages anthropogéniques (culturels). Schreiber (1990) souligne le caractère spatial des études de l'écologie du paysage: elles concernent l'organisation et la répartition des écosystèmes dans le cadre du paysage et l'influence de cette répartition sur le fonctionnement de l'ensemble. D'après Forman (1983), dans ces études il s'agit: a) du paysage dans le sens anthropogénique, b) de son hétérogénéité spatiale, c) des flux entre les unités écologiques (éléments du paysage), d) de l'activité humaine, qui constitue le principal processus dans la plupart des paysages. Enfin, Forman et Gordon (1983) considèrent que trois caractéristiques du paysage doivent être au centre de l'étude:

- sa structure, c'est-à-dire les relations spatiales entre les unités paysagiques (répartition des espèces végétales et animales, ainsi que la distribution de la matière et de l'énergie par rapport à la dimension, la forme, le nombre, la nature et la disposition réciproque des écosystèmes);

- son fonctionnement, c'est-à-dire l'interaction entre les unités spatiales du paysage (le flux de l'énergie et de la matière ainsi que les migrations des organismes entre les écosystèmes);

- sa variabilité, c'est-à-dire les transformations de la structure et des fonctions des unités écologiques.

Les différentes méthodes sont utilisées dans les études de l'écologie du paysage. Certaines sont employées surtout dans la géographie physique, d'autres dans la biologie. Mais dans la plupart des cas les méthodes 
géographiques peuvent être aussi utilisées dans les sciences naturelles qui étudient les êtres vivants (surtout lorsqu'il s'agit de l'analyse spatiale) - et, réciproquement, les méthodes de l'écologie biologique (surtout celles qui concernent la dynamique des phénomènes) peuvent très bien être adoptées dans l'étude du globe terrestre. A l'occasion de la discussion sur la définition de l'écologie du paysage il paraît opportun de souligner les changements dans le sens que l'on donnait au terme "écologie". Il a été introduit en 1869 par Haeckel, biologiste allemand, qui a désigné ainsi la science qui étudie les relations entre un organisme et son environnement organique et minéral. Au début, ce terme n'était utilisé que dans le milieu scientifique, jamais dans le langage courant. Cette perception traditionnelle de l'écologie, bien définie, est toujours admise par la plupart des biologistes et par de nombreux spécialistes d'autres disciplines. En même temps, depuis quelques dizaines d'années, l'écologie signifie aussi les études de sciences naturelles appliquées (ou celles qui se rapportent au milieu naturel), ainsi que toutes les activités qui concernent l'aménagement et la protection du milieu naturel. Dans le langage courant nous avons également vu apparaître de termes ou expressions qui n'ont en général rien à voir avec la notion d'écologie: équilibre écologique, catastrophe écologique (!), technologie écologique, essence écologique, emballage écologique, etc. Ainsi, le terme "écologie" est actuellement très répandu et utilisé avec abus, surtout par les partisans de la protection de la Nature.

Les publications de plus en plus nombreuses témoignent du développement et de la popularité de l'écologie du paysage. Le manuel de Leser Landschaftsökologie, paru en 1969 (dernière édition en 1991), est consacré surtout aux problèmes théoriques et constitue la source principale des connaissances pour les adeptes d'une vue holistique sur la structure du milieu naturel. En 1983, dans Landscape Ecology and Land Use, Vink a insisté sur l'utilité de la théorie des systèmes dans l'étude du milieu naturel. D'après lui, le système dans l'écologie du paysage est un réseau d'objets et/ou de leurs attributs. Des interactions existent entre eux; le tout constitue un ensemble qui a un mode de fonctionnement déterminé. Vink souligne que le paysage doit être traité comme un système contrôlé, car ses composants principaux sont entièrement ou en partie contrôlés par l'homme, lors de l'utilisation et de l'aménagement du terrain.

Un an plus tard parait l'ouvrage bien connu de Naveh et Lieberman (Landscape Ecology. Theory and Application). Ils considèrent que la théorie générale des systèmes, la biocybérnetique et l'écosystémologie constituent la base de l'écologie du paysage. Le rôle actif de l'homme dans le paysage est un de principes de la théorie de l'écologie du paysage. Cela équivaut au refus du dogme de la succession vers le climax. Tuxen a fait le premier pas significatif dans cette direction en présentant les principes de la cartographie de la végétation potentielle. Les mêmes principes apparaissent aussi dans les travaux de Schmithusen sur le paysage naturel potentiel. 
En 1986, Forman et Godron ont publié un ouvrage intitulé également Landscape Ecology; consacré surtout au fonctionnement du milieu naturel, il a un aspect plus biologique.

Le manuel Landscape Ecology - Abstracts of Lectures, édité en 1986 en ex-RDA et préparé par les chercheurs de l'Institut de Géographie et de Géoécologie de Leipzig pour les participants à l'école internationale de l'écologie du paysage, a eu une grande importance pour les pays d'Europe Centrale et Orientale. En 1988, Leser et Klink ont publié un guide de cartographie géoécologique au 1:25 000. L'ouvrage Quantitative Methods in Landscape Ecology, édité en 1991 aux États-Unis sous la rédaction de Turner et Gardner, est consacré à l'analyse de la différenciation et la modélisation du paysage.

La revue Landscape Ecology paraît depuis 1987 sous la rédaction de Golley de l'Institut d'Écologie de l'Université Georgia à Athens (États-Unis). Elle propage le point de vue global de l'étude de la structure et du fonctionnement du paysage, ainsi que des effets de l'activité humaine dans le milieu naturel.

Il est difficile de surestimer le rôle de l'Association internationale de l'écologie du paysage (IALE) dans le développement de l'écologie du paysage dans le monde. Le but de cette organisation, constituée en 1982, est d'approfondir la collaboration entre les chercheurs et praticiens s'occupant de l'environnement, de développer les études interdisciplinaires du milieu naturel, de vulgariser la connaissance du paysage et les possibilités de son utilisation.

En Pologne, les études holistiques du milieu naturel ont une longue tradition. Mentionnons d'abord les travaux de M. Raciborski (1883-1917) et de J.G. Pawlikowski (1860-1939), pionniers du mouvement de la protection de la nature. A. Wodziczko (1887-1947), qui dans ses publications utilisait le terme "biologie du paysage", a développé l'idée holistique de considérer la Nature. J. Smoleński (1881-1940) a élaboré une classification physionomique des paysages, tandis que S. Lencewicz (1889-1944), outre la division de la Pologne en régions naturelles, a distingué six principaux types du paysage naturel, considéré intégralement comme effet de l'interaction de tous les composants de la nature.

Le développement des études intégrées sur le milieu naturel, après la Deuxième Guerre mondiale, a constitué la réponse aux besoins de l'économie. Pour élaborer les bases méthodiques de l'étude du paysage, J. Kondracki, de l'Université de Varsovie, a pris l'initiative d'étudier en détail la structure du milieu naturel en Mazurie et dans le plateau de la Petite Pologne. Les études intégrées du milieu naturel se sont aussi développées en dehors de Varsovie, surtout à Poznań, où T. Bartkowski a créé un centre actif de la géographie du paysage. C'est grâce à son initiative que le groupe de travail de l'IALE "Écologie des villes" a été organisé en 1984, c'est également sous son impulsion que la section polonaise de l'IALE s'est constituée en 1985. Mais, puisque dans la situation politique de l'époque, la constitution d'une nouvelle organisation s'est avérée impossible, les 
membres polonais de l'IALE ont créé un Club de l'Écologie du paysage au sein de l'Association géographique polonaise. Il a organisé plusieurs conférences et séminaires; les actes de ces réunions ont été, dans la plupart des cas, publiés. Ils étaient consacrés à l'importance de l'écologie du paysage dans les activités économiques, à l'étude des régions où l'environnement est particulièrement menacé par ces activités, à la politique économique et sociale dans les terrains transformés sous l'action de l'homme, à la planification du paysage dans les nouvelles conditions sociopolitiques, au fonctionnement et à la valorisation du paysage, à l'agriculture écologique et à l'étude des régions vouées aux loisirs.

L'activité du Club a pris fin en 1993, avec la constitution de l'Association polonaise de l'écologie du paysage. Celle-ci favorisera sans doute une plus grande collaboration entre les naturalistes de diverses formations et le développement des études intégrées du milieu naturel.

\section{BIBLIOGRAPHIE}

Delcourt H.R., Delcourt R.A., 1988, "Quaternary Landscape Ecology: Relevant Scales in Space and Time", Landscape Ecology 2.1.

Form an R.T.T., 1983, LALE. Bulletin 1.1.

Forman R.T.T., Godron M., 1986, Landscape Ecology, S. Wiley and Sons, New York.

Landscape Ecology - Abstracts of Lectures 1986, Leipzig.

Le s e r H., 1991, Landschaftsökologie Ansatz, Modelle, Methodik, Anwendung. Eugen Ulmer Verlag, Stuttgart.

Le ser H., Klink H.J. 1988, Handbuch und Kartierenleitung Geoökologische Karte 1:25 000 (KA GOK 25). Forschungen zur Deutschen Landeskunde BD 228.

Leser H., Rodd M., 1991, "Landscape Ecology - fundamentals, aims and perpectives", In: Es ser G., Overdi e ck O. (eds) Modern Ecology, Elsevier.

Naveh Z., Li berman A., 1984, Landscape Ecology - Theory and Application, Springer Verlag, New York.

Schreiber K.F., 1990, "The History of Landscape Ecology in Europe", In: Z on neveld I.S., Form a n R.T.T. (eds) Changing Landscapes: An Ecological Perpective. Springer Verlag, New York.

Troll C., 1950, Die geographische Landschaft und ihre Erforschung - Studium Generale 3, Arbeiten aus dem Geogr. Inst. der Universität Bonn, 1.5.

Turner M.G., Gardner R.H., (eds), 1991, Quantitative Methods in Landscape Ecology. The Analysis and Interpretation of Landscape Heterogeneity, Springer Verlag.

Vink A.P.A., 1983, Landscape Ecology and Land Use, Longman, London, New York.

Zonneveld J.I.S., 1990, Introduction to "Cultural aspects of Landscape". First Inf. Conf. of the IALE Working group "Culture and Landscape". Ed. H. Svobodova, Wageningen. 
